\title{
Simulation of Thermographic Responses of Delaminations in Composites with Quadrupole Method
}

\author{
William P. Winfree ${ }^{\mathrm{a}}$, Joseph N. Zalameda ${ }^{\mathrm{b}}$, Patricia A. Howell ${ }^{\mathrm{b}}$, and K. Elliott Cramer $^{\mathrm{b}}$ \\ ${ }^{a}$ NASA Langley Research Center, MS 225, Hampton, Va, USA \\ ${ }^{b}$ NASA Langley Research Center, MS 231, Hampton, Va, USA
}

\begin{abstract}
The application of the quadrupole method for simulating thermal responses of delaminations in carbon fiber reinforced epoxy composites materials is presented. The method solves for the flux at the interface containing the delamination. From the interface flux, the temperature at the surface is calculated. While the results presented are for single sided measurements, with flash heating, expansion of the technique to arbitrary temporal flux heating or through transmission measurements is simple. The quadrupole method is shown to have two distinct advantages relative to finite element or finite difference techniques. First, it is straight forward to incorporate arbitrary shaped delaminations into the simulation. Second, the quadrupole method enables calculation of the thermal response at only the times of interest. This, combined with a significant reduction in the number of degrees of freedom for the same simulation quality, results in a reduction of the computation time by at least an order of magnitude. Therefore, it is a more viable technique for model based inversion of thermographic data. Results for simulations of delaminations in composites are presented and compared to measurements and finite element method results.
\end{abstract}

Keywords: thermography, composite, nondestructive evaluation, simulation

\section{INTRODUCTION}

Flash thermography has been shown to be an effective method for rapid inspection of large carbon fiber reinforced polymer (CFRP) composite structures. To better understand the limitations of the technique and enable quantitative characterization of subsurface flaws, it is desirable to simulate the measurement. There are limited analytical or series solutions for the diffusion of heat in a solid and only for simple geometries. ${ }^{1}$ Numerical techniques such as finite difference and finite element methods are well suited for such problems, ${ }^{2,3}$ but can be computationally intensive. Both commercial ${ }^{4-7}$ and noncommercial ${ }^{8,9}$ simulation packages have been successfully applied to simulating thermographic nondestructive evaluation techniques. For simulation of flash heating, some care is required, since the application of short duration flux to the surface results in a large spatial temperature gradient near the surface at early times. To accurately simulate the thermal response, it is necessary to have closely spaced elements near the surface and/or start the simulation with an initial condition that assumes the flux was applied at a fixed time before the start of the simulation, rather than using Neumann boundary conditions to represent a short flux pulse.

Rather than solving the heat equation in the time domain, it is possible to solve the Laplace transform of the heat equation, ${ }^{1}$ then invert the Laplace transform to produce a time domain response. There are a limited number of cases where it is possible to analytically invert the Laplace transform, however it is a very powerful technique for one dimensional multilayered materials where an analytic solution exists for the Laplace transform, which is numerically inverted. This is often much faster and more accurate than finite difference or finite element techniques for the same configuration, since it is possible to solve for only the times of interest and the Laplace transform of impulse flux at the surface is a constant.

Further author information: (Send correspondence to W.P.W.)

W.P.W.: E-mail: william.p.winfree@nasa.gov, Telephone: +1 7568644962

J.N.Z.: E-mail: joseph.n.zalameda@nasa.gov, Telephone: +1 7578644793

P.A.H.: E-mail: p.a.howell@nasa.gov, Telephone: +1 7578644786

K.E.C.: E-mail: k.elliott.cramer@nasa.gov, Telephone: +1 7578647945 
Often, this is referred to as the thermal quadrupole method, and is applicable for modeling responses in materials and structures in general ${ }^{10}$ as well as flash thermography. For flash thermography, it has been used extensively for simulating the thermal response of multilayer systems with and without contact resistances at the interfaces. ${ }^{11-16}$ It has also been shown to be applicable for three dimensional configurations, in particular for delaminations in composites ${ }^{17-19}$ by representing the temperature as a cosine series and solving for the coefficients. This paper follows a similar approach, except the flux is solved for directly and the thermal response is calculated from the flux. It is illustrated by solving for the thermal response of a delamination and comparing that to a finite element solution for the same configuration.

\section{QUADRUPOLE METHOD OF SIMULATING THERMAL RESPONSE OF LAYERS}

A typical application of the quadrupole method is solving the one dimensional heat equation of multilayer systems. For one dimensional problems, a matrix is used to represent the relationship between the temperature and flux of one surface of the layer to the temperature and flux at the other surface (see Fig. 1). If two of the quantities are known (typically the fluxes at the surfaces), then it is possible to solve for the other two. The next subsection illustrates the quadrupole method in one dimension. This facilitates understanding of the extension of the technique to three dimensions.

\subsection{Quadrupole Method in One Dimension}

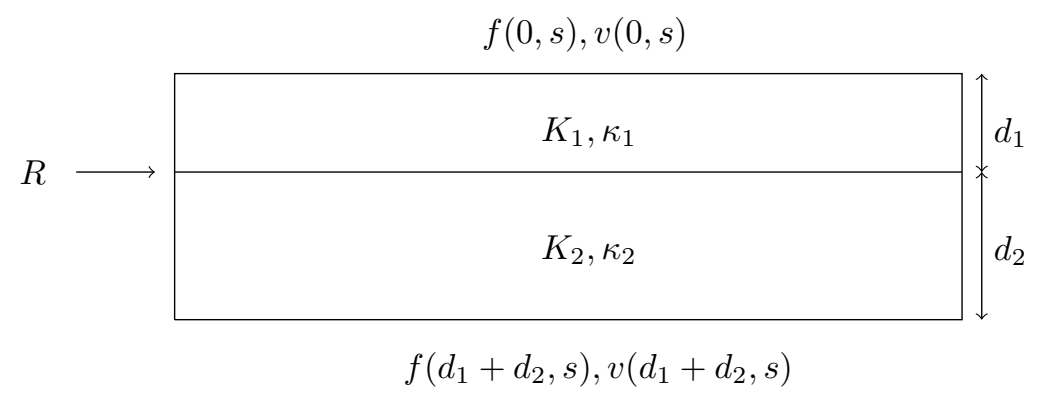

Figure 1. One dimensional configuration for two layer system with a contact resistance between the two layers.

A one dimensional solution for the Laplace transform of temperature in an homogeneous material is ${ }^{1}$

$$
v(z, s)=v(0, s) \cosh \left(z \sqrt{\frac{s}{\kappa}}\right)-f(0, s) \frac{\sinh \left(z \sqrt{\frac{s}{\kappa}}\right)}{K \sqrt{\frac{s}{\kappa}}} .
$$

where $K$ and $\kappa$ are the thermal conductivity and diffusivity, respectively, $v(0, s)$ and $f(0, s)$ are the Laplace transform of temperature and flux at a point, $\mathrm{s}$ is the complex frequency variable and $\mathrm{z}$ is the vertical position. A similar expression for the Laplace transform of the flux as a function of position is

$$
f(z, s)=f(0, s) \cosh \left(z \sqrt{\frac{s}{\kappa}}\right)-v(0, s) \sinh \left(z \sqrt{\frac{s}{\kappa}}\right) K \sqrt{\frac{s}{\kappa}} .
$$

A simple way to express both of the equations is with the matrix formula

$$
\left[\begin{array}{cc}
\cosh (z q) & -\frac{\sinh (z q)}{K q} \\
-K q \sinh (z q) & \cosh (z q)
\end{array}\right]\left[\begin{array}{c}
v(0, s) \\
f(0, s)
\end{array}\right]=\left[\begin{array}{c}
v(z, s) \\
f(z, s)
\end{array}\right]
$$

where $q=\sqrt{\frac{s}{\kappa}}$. From this matrix equation, the Laplace transform of the temperature at the two surfaces can be solved for in terms of the flux at the two surfaces, and is found to be

$$
v(0, s)=\frac{f(0, s) \operatorname{coth}(d q)-f(d, s) \operatorname{csch}(d q)}{K q}
$$


and

$$
v(d, s)=\frac{\operatorname{csch}(d q) f(0, s)-f(d, s) \cosh (d q))}{K q},
$$

where $\mathrm{d}$ is the thickness of the layer. For flash heating at the front $\operatorname{surface}(z=0)$ and an insulated back surface $(z=d)$, the thermal response of the layer can be found by setting $f(0, s)=f_{0}$, which represents impulse heating and $f(d, s)=0$, which can be analytically inverted to give a well known series solution. ${ }^{1}$

The advantage of this representation is obvious when solving for the thermal response of a multiple layer system where each layer is represented by a matrix similar to that given in Equation (3). The matrix is then a transfer matrix, and the combined response of multiple layers is obtained by matrix multiplication. For the system shown in Fig. 1, the contact resistance between layers can also be represented by a matrix as shown in Equation (6)

$$
\left[\begin{array}{cc}
\cosh \left(q_{2} d_{2}\right) & \frac{-\sinh \left(q_{2} d_{2}\right)}{K_{2} q_{2}} \\
-K q_{2} \sinh \left(q_{2} d_{2}\right) & \cosh \left(q_{2} d_{2}\right)
\end{array}\right]\left[\begin{array}{cc}
1 & -R \\
0 & 1
\end{array}\right]\left[\begin{array}{cc}
\cosh \left(q_{1} d_{1}\right) & \frac{-\sinh \left(q_{1} d_{1}\right)}{K_{1} q_{1}} \\
-K q_{1} \sinh \left(q_{1} d_{1}\right) & \cosh \left(q_{1} d_{1}\right)
\end{array}\right]\left[\begin{array}{c}
v(0, s) \\
f(0, s)
\end{array}\right]=\left[\begin{array}{c}
v\left(d_{1}+d_{2}, s\right) \\
f\left(d_{1}+d_{2}, s\right)
\end{array}\right]
$$

where $d_{1}$ and $d_{2}$ are the thicknesses for the first and second layers, $R$ is the contact resistance between the two layers and $q_{n}=\sqrt{s / \kappa_{n}}$. The boundary conditions between the two layers represented by this matrix equation are that the flux across the interface is continuous (energy conservation) and that there is a discontinuity in the temperature proportional to the flux times the contact resistance.

The thermal response of multiple layers can be found by using the same matrix formulation. Assuming that there is a stack of L layers, then the matrix equation becomes

$$
M\left(q_{L}, K_{L}, d_{L}\right) \cdot M\left(q_{L-1}, K_{L-1}, d_{L-1)}\right) \cdots M\left(q_{2}, K_{2}, d_{2)}\right) \cdot M\left(q_{1}, K_{1}, d_{1)}\right) \cdot\left[\begin{array}{c}
v(0, s) \\
f(0, s)
\end{array}\right]=\left[\begin{array}{c}
v(D, s) \\
f(D, s)
\end{array}\right],
$$

where $\mathrm{D}$ is the thickness of the total stack and

$$
M\left(q_{l}, K_{l}, d_{l}\right)=\left[\begin{array}{cc}
\cosh \left(q_{l} d_{l}\right) & \frac{-\sinh \left(q_{l} d_{l}\right)}{K_{l} q_{l}} \\
-K_{l} q_{l} \sinh \left(q_{l} d_{l}\right) & \cosh \left(q_{l} d_{l}\right)
\end{array}\right]
$$

If for one of the layers $q_{l} d_{l}$ is small, then $M\left(q_{l}, K_{l}, d_{l}\right)$ reduces to approximately the same form as the middle matrix in Equation (6), with the substitution of $d_{n} / K_{n}$ for the contact resistance(R). By performing all of the matrix multiplications, one obtains a single matrix $T(D, s)$ given by

$\mathbf{T}(D, s)=\left[\begin{array}{ll}T_{1,1}(D, s) & T_{1,2}(D, s) \\ T_{2,1}(D, s) & T_{2,2}(D, s)\end{array}\right]=M\left(q_{L}, K_{L}, d_{L}\right) \cdot M\left(q_{L-1}, K_{L-1}, d_{L-1)}\right) \cdots M\left(q_{2}, K_{2}, d_{2}\right) \cdot M\left(q_{1}, L_{1}, d_{1)}\right)$.

If the flux at the top of the stack and the bottom of the stack are known, then the temperatures at those locations are given by

$$
v(0, s)=\frac{f(D, s)-f(0, s) T_{2,2}(D, s)}{T_{2,1}(D, s)}
$$

and

$$
v(D, s)=\frac{f(D, s) T_{1,1}(D, s)+f(0, s)\left(T_{1,2}(D, s) T_{2,1}(D, s)-T_{1,1}(D, s) T_{2,2}(D, s)\right)}{\left.T_{2,1}(D, s)\right)} .
$$

The thermal response at a single time is found at either surface numerically by using Eqs. (10) or (11) in a numerical inverse Laplace transform algorithm. 


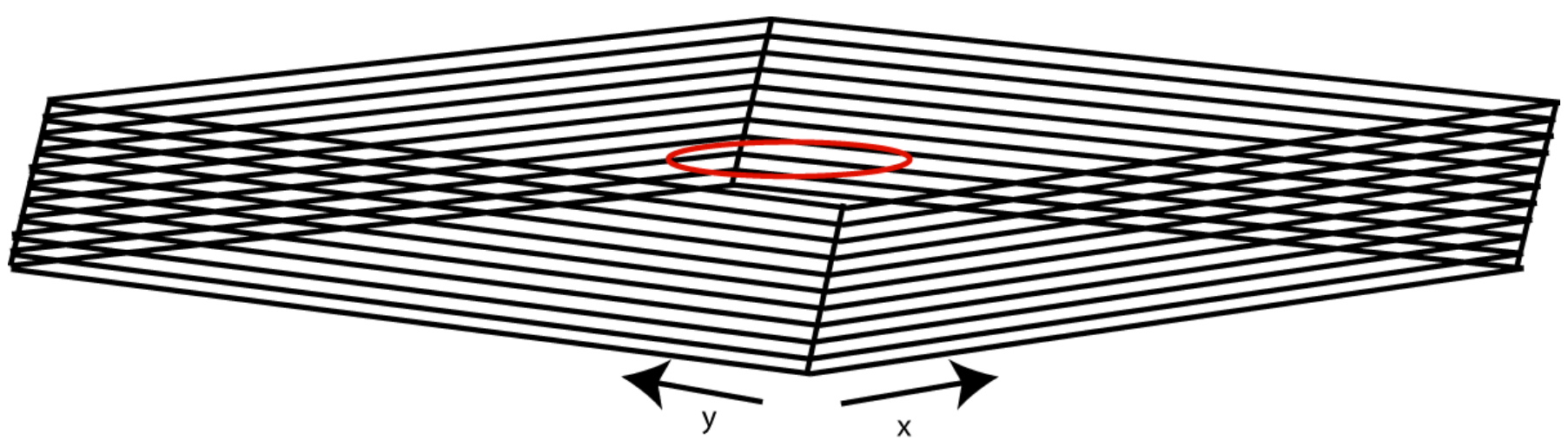

Figure 2. Three dimensional configuration for multilayer system with a circular delamination that reduces flux between two of the layers.

\subsection{Quadrupole Method in Three Dimensions}

Assuming a homogeneous layer with finite lateral dimensions, as is shown in Fig. 2, with no heat flow across the vertical edges at $x=0, y=0, x=L_{x}$ and $y=L_{y}$, a solution to the Laplace transform of the heat equation in the layer can be represented by the expression

$$
v(x, y, z, s)=\sum_{m=0}^{M-1} \sum_{n=0}^{N-1} a_{m} a_{n} \tilde{v}_{m, n}(z, s) \cos \left(\frac{\pi x m}{L_{x}}\right) \cos \left(\frac{\pi y n}{L_{y}}\right),
$$

where $L_{x}$ and $L_{y}$ are the lateral width of the layer in the $x$ and $y$ directions, respectively, and $a_{m}=1$ if $m$ is equal to 0 or $M-1$, respectively, otherwise $a_{m}=2$ and the cosine series coefficient is $\tilde{v}_{m, n}(z, s)$ given by

$$
\tilde{v}_{m, n}(z, s)=\frac{\sum_{i=0}^{M-1} \sum_{j=0}^{N-1} a_{i} a_{j} v\left(x_{i}, y_{j}, z, s\right) \cos \left(\frac{\pi x_{i} m}{L_{x}}\right) \cos \left(\frac{\pi y_{j} m}{L_{y}}\right)}{(2 M-2)(2 N-2)}
$$

or

$$
\tilde{v}_{m, n}(z, s)=\frac{\sum_{i=0}^{M-1} \sum_{j=0}^{N-1} a_{i} a_{j} v_{i, j}(z, s) \cos \left(\frac{\pi m i}{M-1}\right) \cos \left(\frac{\pi n j}{N-1}\right)}{(2 M-2)(2 N-2)},
$$

where the temperature is defined at a set of $\mathrm{M}$ by $\mathrm{N}$ evenly spaced locations given by $x_{m}=m L_{x} /(M-1)$ and $y_{n}=n L_{y} /(N-1)$. The flux in the layer at the same $\mathrm{x}$ and $\mathrm{y}$ locations is given by a similar expression

$$
f_{m, n}(z, s)=\frac{\sum_{i=0}^{M-1} \sum_{j=0}^{N-1} a_{m} a_{n} \tilde{f}_{i, j}(z, s) \cos \left(\frac{\pi i m}{2 M-2}\right) \cos \left(\frac{\pi j n}{2 N-2}\right)}{(2 M-2)(2 N-2)},
$$

where $\tilde{f}_{m, n}(z, s)$ is also given by Equation (14), if $f$ is substituted for $v$. Within each layer, Equation (12) is a solution to the Laplace transform of the heat equation if the cosine series coefficients for the temperature have a $z$ dependence given by

$$
\tilde{v}_{m, n}(z, s)=\tilde{v}_{m . n}(0, s) \cosh \left(z q_{m, n}\right)-\tilde{f}_{m, n}(0, s) \frac{\sinh \left(z q_{m, n}\right)}{K q_{m, n}},
$$

where $q_{m, n}$ is given by

$$
q_{m, n}=\sqrt{\frac{s}{\kappa_{z}}+\frac{\kappa_{x}}{\kappa_{z}}\left(\frac{\pi m}{L_{x}}\right)^{2}+\frac{\kappa_{y}}{\kappa_{z}}\left(\frac{\pi n}{L_{y}}\right)^{2}}
$$


This is similar to the one dimensional equation for the $\mathrm{z}$ dependence of the Laplace transform (Equation (1)) and the $z$ dependence of flux similar to Equation (2). There is also a simple matrix equation similar to Equation (3) for each spatial frequency term in Equation (14),

$$
\left[\begin{array}{cc}
\cosh \left(z q_{m, n}\right) & -\frac{\sinh \left(z q_{m, n}\right)}{K q_{m, n}} \\
-K q_{m, n} \sinh \left(z q_{m, n}\right) & \cosh \left(z q_{m, n}\right)
\end{array}\right]\left[\begin{array}{c}
\tilde{v}_{m, n}(0, s) \\
\tilde{f}_{m, n}(0, s)
\end{array}\right]=\left[\begin{array}{c}
\tilde{v}_{m, n}(z, s) \\
\tilde{f}_{m, n}(z, s)
\end{array}\right] .
$$

If there is a stack of layers with no discontinuities at the interfaces between layer, then it it possible to use equations with an equivalent form to Eqs (10) and (11), if $\mathbf{T}(m, n, D, s)$ is found using the appropriate $q_{m, n}$ for each layer or

$$
\tilde{v}_{m, n}(0, s)=\frac{\tilde{f}_{m, n}(D, s)-\tilde{f}_{m, n}(0, s) T_{2,2}(m, n, D, s)}{T_{2,1}(m, n, D, s)}
$$

and

$\tilde{v}_{m, n}(D, s)=\frac{\tilde{f}_{m, n}(D, s) T_{1,1}(m, n, D, s)+\tilde{f}_{m, n}(0, s)\left(T_{1,2}(m, n, D, s) T_{2,1}(m, n, D, s)-T_{1,1}(m, n, D, s) T_{2,2}(m, n, D, s)\right)}{\left.T_{2,1}(m, n, D, s)\right)}$.

If there is a spatially varying contact resistance at the interface between two layers in the $\operatorname{stack}\left(z=d_{1}\right)$, then Eqs. (19) and (20) are no longer correct for the whole stack. However, if one divides the stack into two stacks, then it is possible to use Eqs. (19) and (20) to find the temperatures at the top and bottom of each stack if the cosine series coefficients are known for the flux at the interface with the spatially varying contact resistance.

Dividing the total stack into two separate stacks, one $d_{1}$ thick and the other $d_{2}$ thick, separated by an interface with the spatially varying contact resistance, the object is to find the flux at that interface. It is possible to calculate the cosine series coefficient for the Laplace transform of the temperature above that interface from $\tilde{f}_{m, n}(0)$ and $\tilde{f}_{m, n}\left(d_{1}\right)$ from Equation $(20)$ and the coefficients for the temperature below the interface from $\tilde{f}_{m, n}\left(d_{1}\right)$ and $\tilde{f}_{m, n}(D)$ using Equation (19).

The Laplace transform of the temperature above the interface, in terms of the flux at the interface (the flux is continuous across the interface) is found using Eqs. (12), (14) and (20), the Laplace transform of the temperature above the interface at discrete $(x, y)$ positions can be represented in terms of the Laplace transform of the fluxes at $z=0$ and $z=d_{1}$ by

$$
v_{i, j}^{+}\left(d_{1}, s\right)=\sum_{m=0}^{M-1} \sum_{n=0}^{N-1} C_{i, j, m, n}^{1}\left(d_{1}, s\right) f_{m, n}(0, s)-C_{i, j, m, n}^{2}\left(d_{1}, s\right) f_{m, n}\left(d_{1}, s\right),
$$

where

$$
\begin{aligned}
C_{i, j, m, n}^{1}(d, s) & =a_{m} a_{n} \frac{\sum_{p=0}^{M-1} \sum_{q=0}^{N-1} a_{p} a_{q} \cos \left(\frac{i p \pi}{M-1}\right) \cos \left(\frac{m p \pi}{M-1}\right) \cos \left(\frac{j q \pi}{N-1}\right) \cos \left(\frac{n q \pi}{N-1}\right) \frac{T_{2,2}(m, n, d, s)}{T_{2,1}(m, n, d, s)}}{(2 M-2)(2 N-2)}, \\
C_{i, j, m, n}^{2}(d, s) & =a_{m} a_{n} \frac{\sum_{p=0}^{M-1} \sum_{q=0}^{N-1} a_{p} a_{q} \cos \left(\frac{i p \pi}{M-1}\right) \cos \left(\frac{m p \pi}{M-1}\right) \cos \left(\frac{j q \pi}{N-1}\right) \cos \left(\frac{n q \pi}{N-1}\right) \frac{1}{T_{2,1}(m, n, d, s)}}{(2 M-2)(2 N-2)},
\end{aligned}
$$

and the $\mathbf{T}(d, s)$ represents the transfer matrix for a stack $d$ thick. By representing the Laplace transform of temperatures and fluxes as vectors, then the system of equations for the Laplace transform of temperature above the interface can be represented as

$$
\mathbf{V}^{+}\left(d_{1}, s\right)=\mathbf{C}^{1}\left(d_{1}, s\right) \cdot \mathbf{F}(0, s)-\mathbf{C}^{2}\left(d_{1}, s\right) \cdot \mathbf{F}\left(d_{1}, s\right)
$$

Assuming the flux at the back surface is zero, a similar systems of equations for below the interface is represented by

$$
\mathbf{V}^{-}\left(d_{1}, s\right)=\mathbf{C}^{2}\left(d_{2}, s\right) \cdot \mathbf{F}\left(d_{1}, s\right),
$$


where the elements of the matrices are defined by Eqs. (22) and (23).

The contact resistance at the interface can also be represented as a matrix $(\boldsymbol{R})$ with the diagonal values equal to the contact resistances at each point in the interface. The boundary conditions at locations at the interface are that the flux is continuous and the temperature difference is the flux times the contact resistance. Using the same matrix representation

$$
\mathbf{V}^{+}\left(d_{1}, s\right)=\mathbf{V}^{-}\left(d_{1}, s\right)+\mathbf{R} \cdot \mathbf{F}\left(d_{1}, s\right) .
$$

Substituting the values for $\mathbf{V}^{+}\left(d_{1}, s\right)$ and $\mathbf{V}^{-}\left(d_{1}, s\right)$ from Eqs. (24) and (25) into Equation (26), one gets

$$
\left.\left(\mathbf{C}^{2}\left(d_{1}, s\right)+\mathbf{C}^{2}\left(d_{2}, s\right)+\mathbf{R}\right) \cdot \mathbf{F}^{(} d_{1}, s\right)=\mathbf{C}^{1}\left(d_{1}, s\right) \cdot \mathbf{F}(0, s)
$$

which can be used to solve for $\mathbf{F}\left(d_{1}, s\right)$ by one of many numerical techniques if the flux at $z=0$ is known.

With the flux at the interface calculated, it is possible to calculate the Laplace transform of the temperature at the surface of interest $(z=0)$ from the expression

$$
\mathbf{V}(0, s)=\mathbf{C}^{2}\left(d_{1}, s\right) \cdot \mathbf{F}(0, s)-\mathbf{C}^{1}\left(d_{1}, s\right) \cdot \mathbf{F}\left(d_{1}, s\right) .
$$

To calculate the temporal response, the Laplace transform is numerically inverted using the Talbot numerical inversion of the Laplace transform which has given sufficient accuracy for simulations performed in the past. ${ }^{13,16}$ The Tablot method uses the trapezoidal rule to numerically invert the Laplace transform at a given time. For this effort, calculating the Laplace transform at only six values of complex frequency $(s)$ gives sufficiently accurate results.

\section{QUADRAPOLE SIMULATIONS OF DELAMINATIONS IN COMPOSITES}

\subsection{Comparison of Quadrupole Method and Finite Element Method Results}

The thermal responses for a delamination were estimated from the quadrupole and compared to the responses estimated from a finite element method software package. A composite was simulated with 10 layers, each 0.019 $\mathrm{cm}$ thick. The thermal conductivity of the layer was assumed to be $0.97 \mathrm{~W} / \mathrm{m} /{ }^{\circ} \mathrm{K}$ in the $\mathrm{z}$ direction (perpendicular to the plane of the plies) and within the plies perpendicular to the fiber direction and $9.7 \mathrm{~W} / \mathrm{m} /{ }^{\circ} \mathrm{K}$ in the direction of the fibers. A $(0,90,0,90,0)_{s}$ ply layup was simulated with the 0 angle being in the x-direction. The density and heat capacity of all the plies was set to be the same and equal to $1600 \mathrm{~kg} / \mathrm{m}^{3}$ and $1200 \mathrm{~J} / \mathrm{kg} /{ }^{\circ} \mathrm{K}$, respectively. The $\mathrm{x}$ and $\mathrm{y}$ dimension of the composite were set to be $1 \mathrm{~cm}$ and the size of the circular delamination was set to $0.25 \mathrm{~cm}$ in diameter. The results are shown in Fig. 3.

The circular delaminations were set at depths of one, two, three and four plies. The time of comparison for equal configuration was chosen to be $d_{1}^{2} / \kappa_{z z} / 4$, where $d_{1}$ is the depth of the delamination, $\kappa_{z}$ is the diffusivity in the direction perpendicular to the surface. This corresponds to the time when the thermal response to flash heating of a layer $d_{1}$ thick has a response approximately 1.037 times the response of a semi-infinite solid of the material. For the delamination one, two, three and four plies deep, this corresponds to times of 0.0166 sec, $0.0694 \mathrm{sec}, 0.1573 \mathrm{sec}$ and $0.2805 \mathrm{sec}$, respectively. All of the responses were normalized by multiplying by $K_{z} \sqrt{\left(\pi t / \kappa_{z}\right)} / F$ where $\mathrm{t}$ is the time of the response and $\mathrm{F}$ is the instantaneous flux. For a semi-infinite solid, this results in a value of 1 for all times. All of the response are scaled from 0.99 to 1.04.

As can be seen from the figure, the FEM and quadrupole results are qualitatively the same. The amplitude of the response decreases as the depth below the surface increases as a result of the in-plane heat diffusion. If there was no in-plane heat diffusion, then the normalization results in the indication amplitudes being approximately equal to $1+2 e^{-4}$ or 1.037 . The only clear indication of the higher conductivity in the $\mathrm{x}$ direction for the first ply is for the delamination just below the first ply. One can see from the figure, the size of the response is wider in the $\mathrm{x}$ direction than in the $\mathrm{y}$ direction. This is also the case for the delamination under three plies, however it is not so pronounced. 


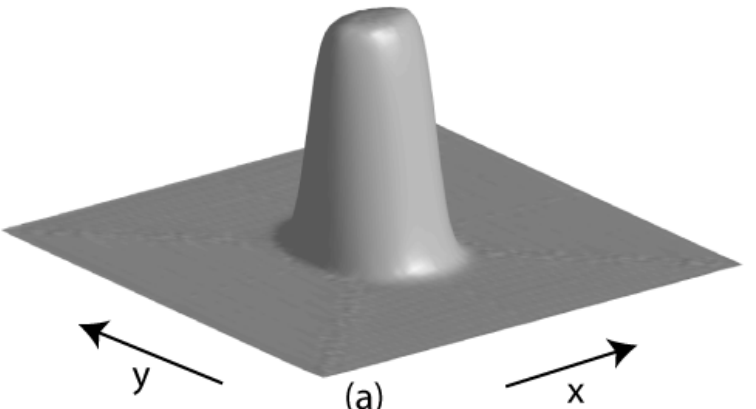

(a)

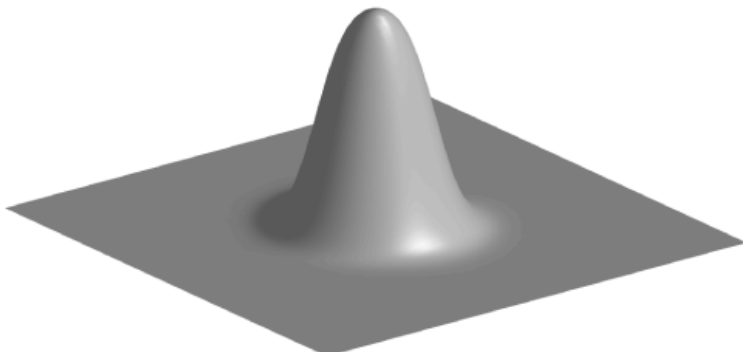

(c)

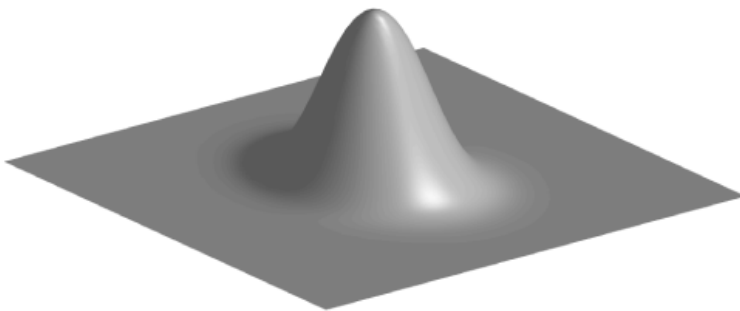

(e)

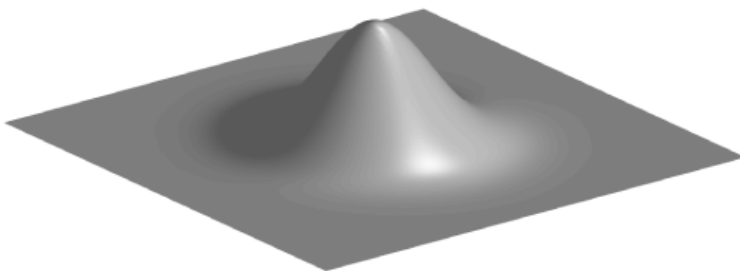

(g)

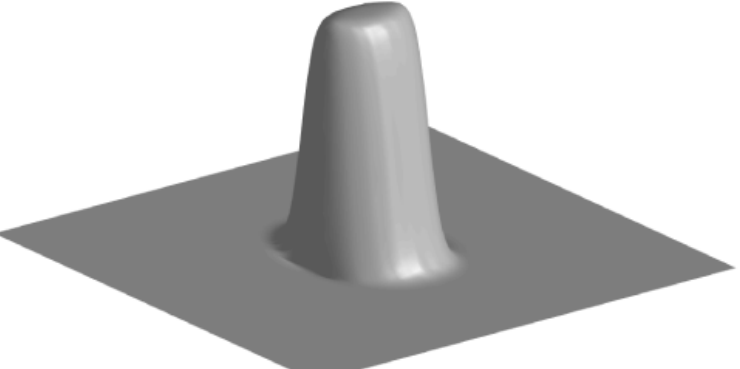

(b)

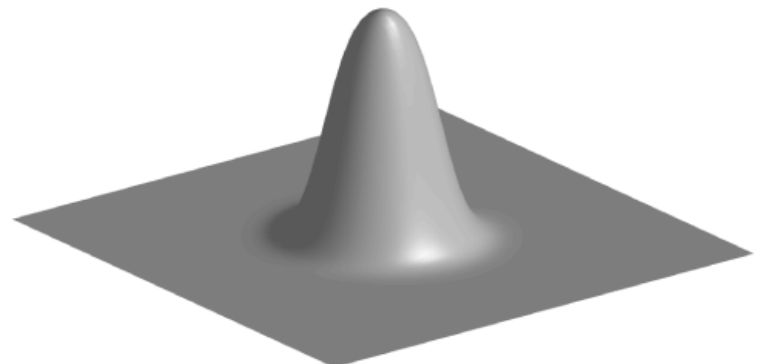

(d)

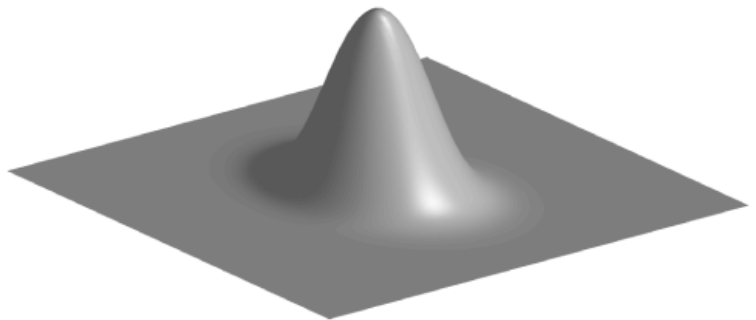

(f)

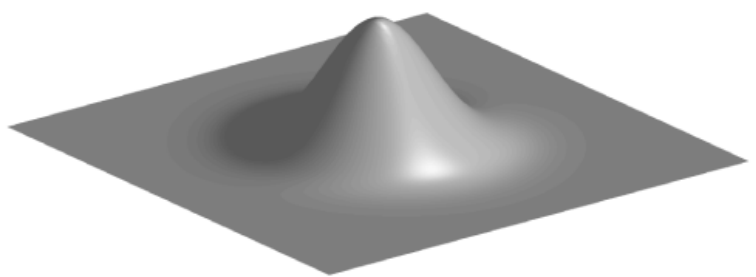

(h)

Figure 3. The thermal response calculated from the quadrupole (b,d,f,h) and finite element method (a,c,e,g) for delaminations one ply (a and b), two plies (c and d), three plies (e and f) and four plies ( $\mathrm{g}$ and $\mathrm{h}$ ) deep.

A quantitative comparison can be seen in the temperature profiles across the center of the delamination shown in Fig. 4. With the normalization, the temperature at position 0 should be one. As can be seen from the figure for both the FEM and quadrupole results, this is not quite the case. It is possible to get closer to one by doing finer vertical grid spacing in the heated surface for the FEM solution and by solving for more values of $s$ for the quadrupole solution. For the quadrupole method, the solutions are less than $0.1 \%$ greater than one, which is much better than experimental data (solving at 8 values for $s$, instead of 6 , does reduce the difference to less than $0.01 \%$ ). All of the FEM solutions required 30 minutes or more of computational time, therefore increasing the density of grid points was not attempted. 


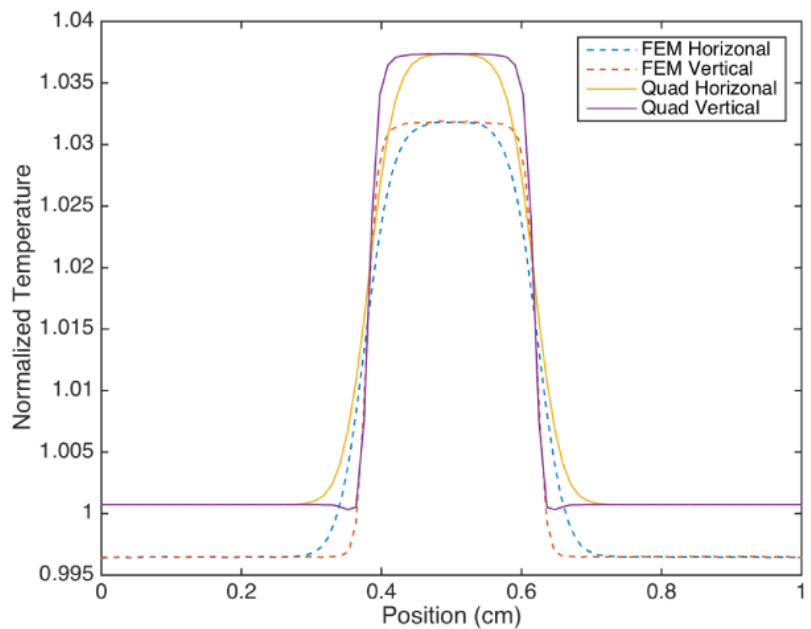

(a)

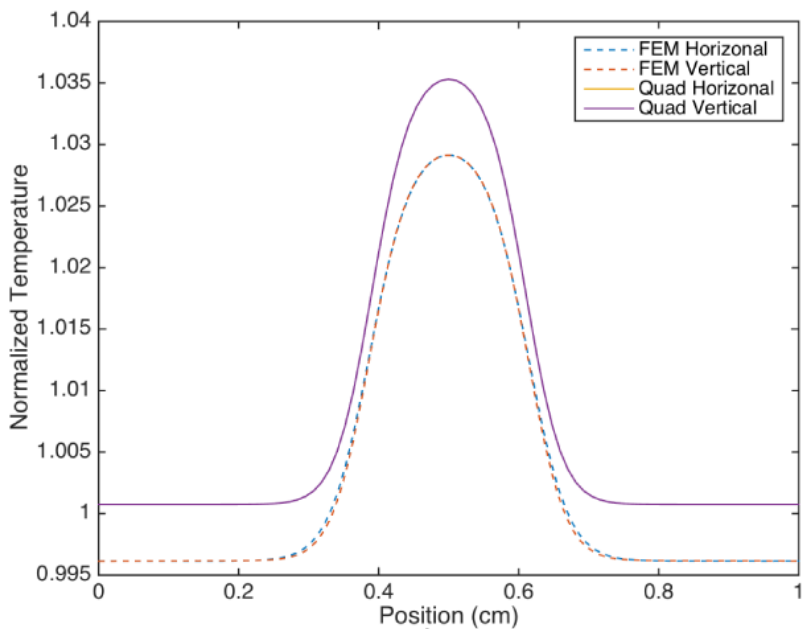

(b)

Figure 4. Temperature profiles across the center of the delamination in the horizontal direction (fiber direction for first ply) and vertical direction for delamination (a) one ply down and (b) two plies down.

As can be seen from the figure, if one divides the profiles by the value of each profile at position 0 to normalize the data, the FEM and quadrupole are in good agreement. Both show that there is only significantly more heat diffusion in a preferred direction when the delamination is under the first ply (the fiber direction in the first ply). An examination of the response for deeper delaminations indicates that while there is preferential diffusion when there is a greater number of plies above the delamination having the same fiber direction orientation, it is not as pronounced as it is for this case. A small interesting characteristic which appears in the quadrupole method is the dip in temperature at the edge of the delamination for the profile running perpendicular to the first ply fiber direction for the case of a delamination under the first ply. This is a result of the fibers in the second ply being oriented in a direction perpendicular to the fibers in first ply. The size of the dip can be increased or decreased by increasing or decreasing the thermal conductivity in the fiber direction.

Finally, the quadrupole simulations require considerably less computational time than the commercial finite element method. The computational time depends on the specifics of the simulation, however, each of the quadrupole simulations were performed in less than two minutes and all of the finite element method solutions required thirty minutes or more on a MacBook Pro.

\subsection{Quadrupole Method Estimate of Thermal Response of Realistic Shaped Damage}

One of the advantages of the quadrupole method is the relative ease with which realistic shapes can be included in the simulation. Figure 5 is the shape of a delamination estimated from the x-ray computed tomography data on a composite specimen with impact damage. At each point in the damage, a contact resistance of $1{ }^{\circ} \mathrm{K} \mathrm{m} / \mathrm{W}$ is used to represent the delamination. This is very large contact resistance (equivalent to about $2 \mathrm{~cm}$ of air), that was intended to reduce the heat flow across the delaminated area to approximately zero. The composite size was 1.5 by $1.36 \mathrm{~cm}$.

The composite thermal properties and thickness of the plies were set to be the same as values as in the previous simulation. One set of simulations was performed with the first ply having a horizontal fiber direction and the second set with the first ply with a vertical fiber direction, with the alternating fiber directions for each subsequent ply. If zero degrees is considered to be horizontal, this is equivalent to $[0,90,0,90,0]_{s}$ and $[90,0,90,0,90]_{s}$ for the two different configurations. For both configurations, the flaw shape shown in Fig. 5 was placed under the first, second, third and fourth plies.

The quadrupole simulation results for the realistically shaped flaw are show in Fig. 6. For the delamination one, two, three and four plies deep, the times of for the simulated images are $0.0166 \mathrm{sec}, 0.0694 \mathrm{sec}, 0.1573 \mathrm{sec}$ 


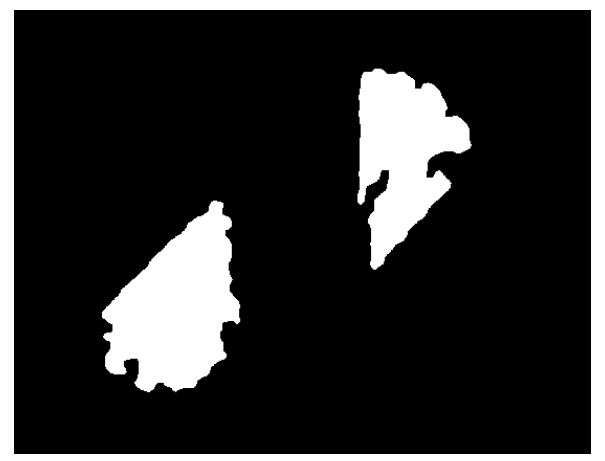

Figure 5. Estimated shape of a delamination from x-ray computed tomography data. The delamination was generated by impacting the composite with a small projectile.

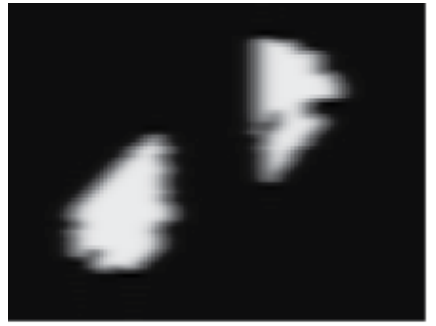

(a)

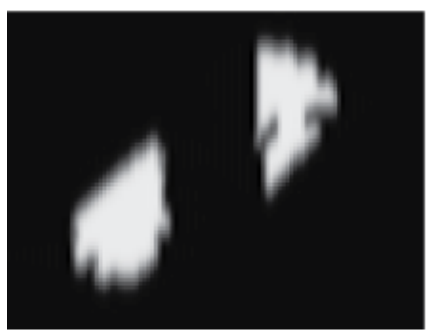

(e)

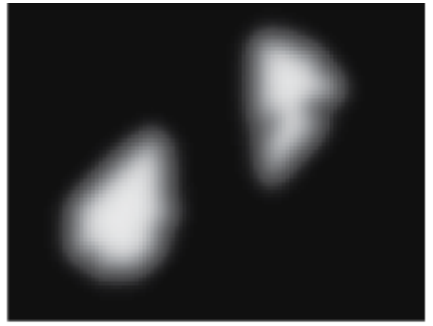

(b)

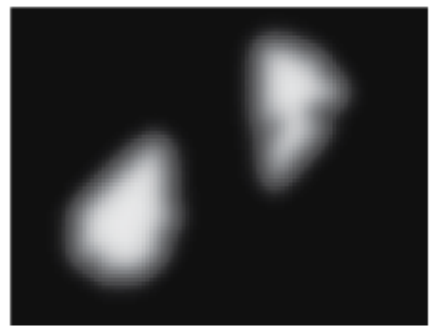

(f)

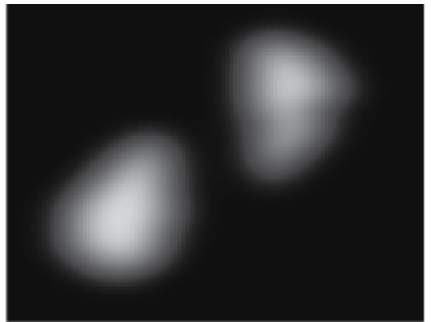

(c)

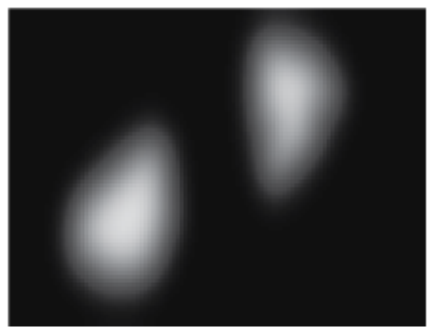

(g)

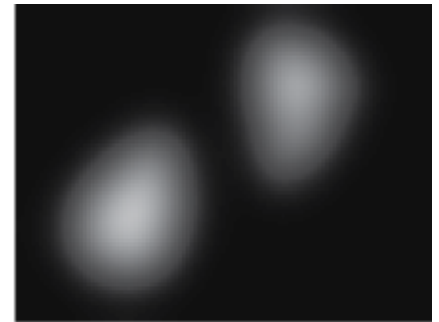

(d)

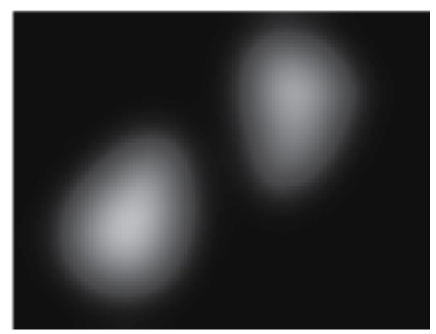

(h)

Figure 6 . The thermal response calculated by the quadrupole method for a realistically shaped delamination in a composite with a $[0,90,0,90,0]_{s}(\mathrm{a}-\mathrm{d})$ and $[90,0,90,0,90]_{s}$ (e-h) ply layup. For (a) and (e) the delamination is one ply down. For (b) and (f) the delamination is two plies down. For (c) and (g) the delamination is three plies down. For (d) and (h) the delamination is four plies down. 
and $0.2805 \mathrm{sec}$, respectively (corresponding to $d^{2} / \kappa_{z z} / 4$ where $d$ is the depth of the flaw). All of the thermal responses are normalized and then scaled from 1 to 1.04. For the flaw under the first ply(a and e), the shape of the thermal response captures the shape of the flaw reasonably well. As would be expected, the shape becomes more and more blurred as the flaw is placed deeper, with general shape being visible when the flaw is four plies down, however all of the details have been lost.

It is possible to see the increase blurring in the horizontal or vertical directions in the fiber direction when the flaw is directly under the first ply. For the cases of the flaw two and four plies down (b and f, $d$ and $h$ ), with the same number of plies with fibers running in the vertical and horizontal directions, the results are very similar for both ply orientations. For three plies above the flaw (c and g), the thermal response is a little wider in the direction of the majority fiber direction.

\section{COMPARISON OF QUADRUPOLE SIMULATION RESULTS TO MEASURED THERMAL RESPONSE OF COMPOSITE WITH FLAT BOTTOM HOLES}

\subsection{System for Measurement of Thermal Response}

The thermal response of the composite specimen was acquired with a commercial flash thermographic measurement system. ${ }^{20,21}$ The front surface of the composite is heated with a flash lamp. The flash duration has been measured to be approximately 0.008 second. Since the earliest thermal responses of interest occur approximately one tenth of a second after the heat pulse, this is a good estimate of the impulse excitation. The thermal response was measured with a focal plane array infrared imager detector with an array size of $640 \times 512$ that operates in the 3-5 micrometer wavelength band. The approximate spatial resolution of the acquired thermography images was $0.04 \mathrm{~cm}$. The imager digital output frame rate was $60 \mathrm{hertz}$ and was connected to a real time digital image processor to acquire the output images. All of the data of interest were acquired within 2.5 seconds after the flash heating.

\subsection{Composite Flat Bottom Hole Specimen}

The composite specimen was approximately $10 \mathrm{~cm} \times 10 \mathrm{~cm}$ with a thickness of $0.212 \mathrm{~cm}$. Nine approximate flat bottom holes were drilled in from the back side of the specimen with depths of approximately one fourth, one half and three fourths of the thickness, and diameters of approximately $1.27 \mathrm{~cm}, 0.63 \mathrm{~cm}$ and $0.32 \mathrm{~cm}$. More accurate measurements of the depth and diameters were determined from x-ray computed tomography data shown in Fig. 7 and are given in Tab. 1. For comparison, an early time thermal response is shown in the same figure.

As can be seen from the computed tomography data in Fig. 7, the top of the flat bottom holes are significantly deeper in the center. The sizes and depths of the holes as measured from the computed tomography data are shown in Tab. 1. Based on the standard deviations of the depth, the holes with a diameter of approximately $0.64 \mathrm{~cm}$ have the flattest tops and the largest holes have the least flat tops. The deeper the hole was drilled the less flat the top of the hole. This lack of flatness is reflected in the thermal response shown as Fig. 7. In the thermal image it is clear that the slight increase in material at the center of the largest hole results in a slightly cooler location at the center of the hole response. Measurements at later times have this same cool spot at the center of all the largest holes, however, it is not as significant for the shallower holes.

\subsection{Simulations of Composite Flat Bottom Hole Specimen}

While flat bottom holes have slightly different responses than the delaminations, they are significantly easier to fabricate than specimens with delaminations. The quadrupole method was used to simulate the thermal responses of the flat bottom holes 0.32 and $0.64 \mathrm{~cm}$ in diameter and approximately 0.05 and $0.1 \mathrm{~cm}$ below the surface. The thermal properties where the same as used in section 3, with the exception of the thermal conductivity in the fiber direction, where $10.4 \mathrm{~W} / \mathrm{m} /{ }^{\circ} \mathrm{K}$ was found to give better agreement between the simulation and the measured responses. The orientation of the fiber direction was alternated with each ply with $0^{\circ}$ for the first ply and 22 plies were in the layup. The ply thickness was set based on the depth of the flat bottom hole and was set to the depth of the hole divided by 5 for the holes approximately $0.05 \mathrm{~cm}$ below the surface and divided by 11 for holes approximately $0.105 \mathrm{~cm}$ below the surface. This results in the hole top always being at a ply interface. 


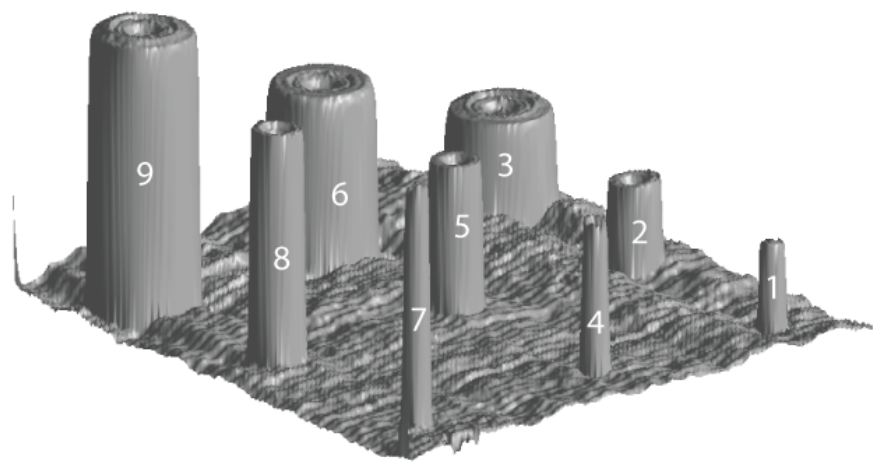

(a)

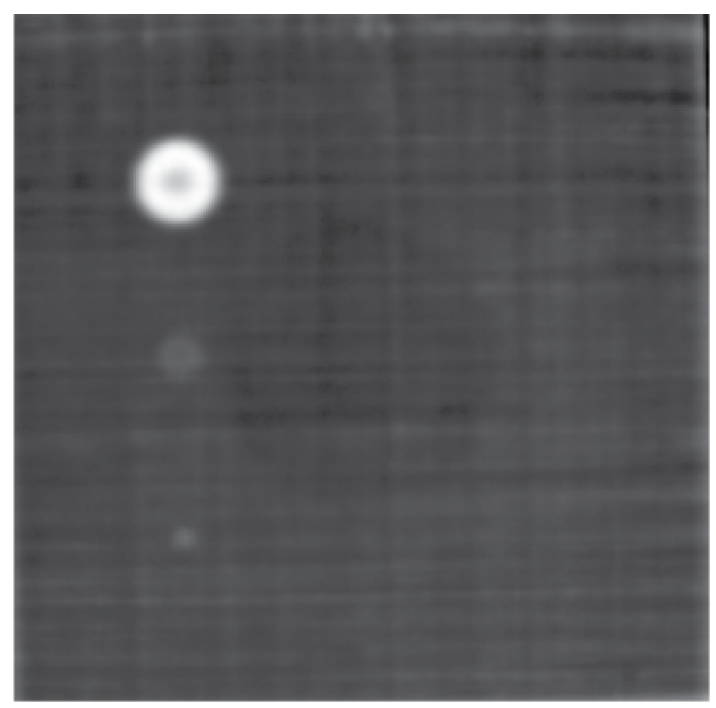

(b)

Figure 7. Computed tomography and thermal response of composite specimen. (a) Representation of the x-ray computed tomography data acquired on composite flat bottom hole specimen. (b) The measured thermal response from specimen acquired 0.25 seconds after the flash heating.

Table 1. Size and Depth of Holes in Composite Flat Bottom Hole Specimen Based on the Computed Tomography Data

\begin{tabular}{|c|c|c|c|}
\hline Hole Number & $\begin{array}{c}\text { Depth From } \\
\text { Front Surface } \\
\text { Above Hole }\end{array}$ & Percent Thickness & Diameter \\
\hline 1 & $0.154 \pm 0.003 \mathrm{~cm}$ & $73 \%$ & $0.32 \pm 0.01 \mathrm{~cm}$ \\
2 & $0.156 \pm 0.003 \mathrm{~cm}$ & $74 \%$ & $0.64 \pm 0.01 \mathrm{~cm}$ \\
3 & $0.148 \pm 0.005 \mathrm{~cm}$ & $70 \%$ & $1.28 \pm 0.01 \mathrm{~cm}$ \\
4 & $0.105 \pm 0.008 \mathrm{~cm}$ & $50 \%$ & $0.32 \pm 0.01 \mathrm{~cm}$ \\
5 & $0.109 \pm 0.004 \mathrm{~cm}$ & $51 \%$ & $0.64 \pm 0.01 \mathrm{~cm}$ \\
6 & $0.097 \pm 0.008 \mathrm{~cm}$ & $46 \%$ & $1.29 \pm 0.01 \mathrm{~cm}$ \\
7 & $0.051 \pm 0.019 \mathrm{~cm}$ & $24 \%$ & $0.32 \pm 0.01 \mathrm{~cm}$ \\
8 & $0.053 \pm 0.007 \mathrm{~cm}$ & $25 \%$ & $0.64 \pm 0.01 \mathrm{~cm}$ \\
9 & $0.027 \pm 0.014 \mathrm{~cm}$ & $13 \%$ & $1.28 \pm 0.01 \mathrm{~cm}$ \\
\hline
\end{tabular}




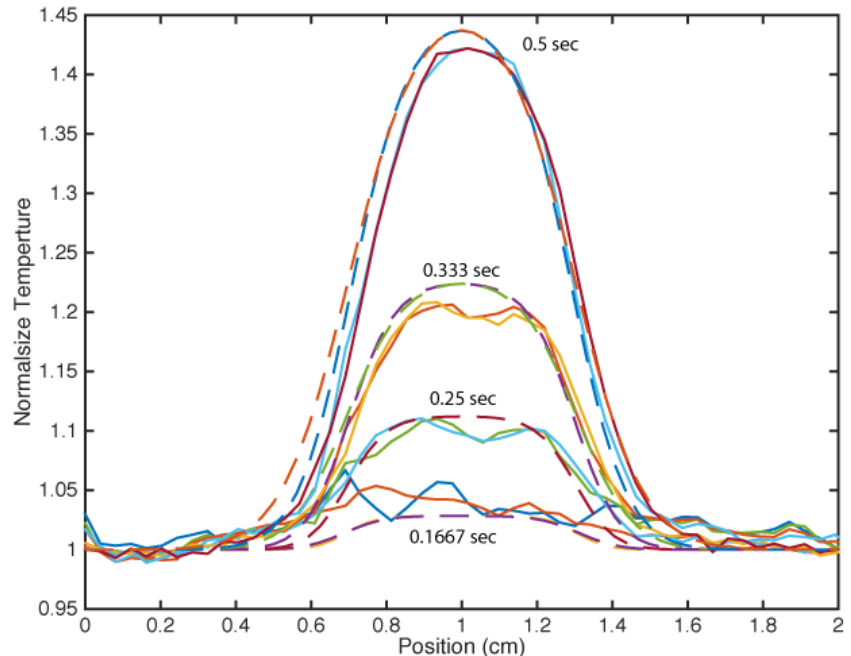

(a)

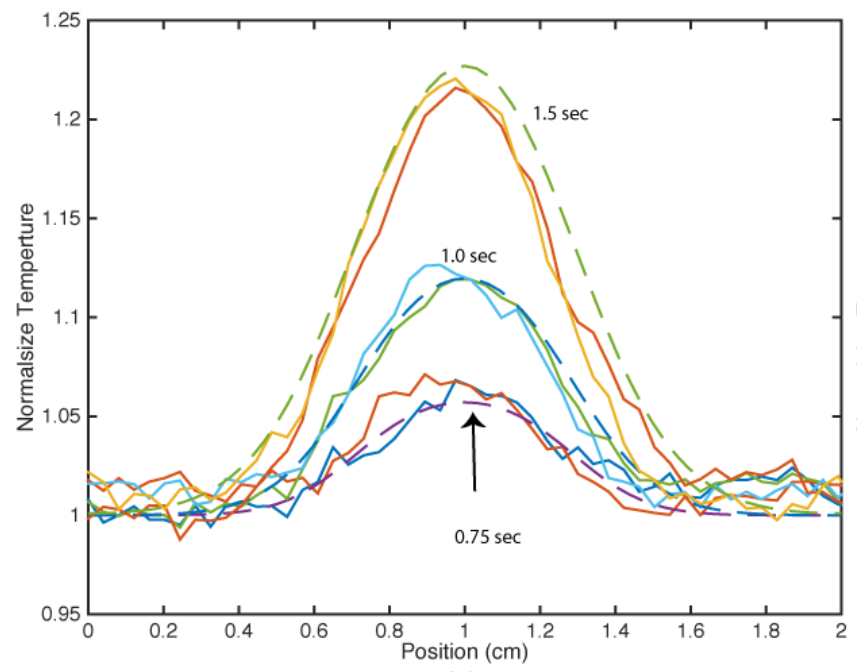

(c)

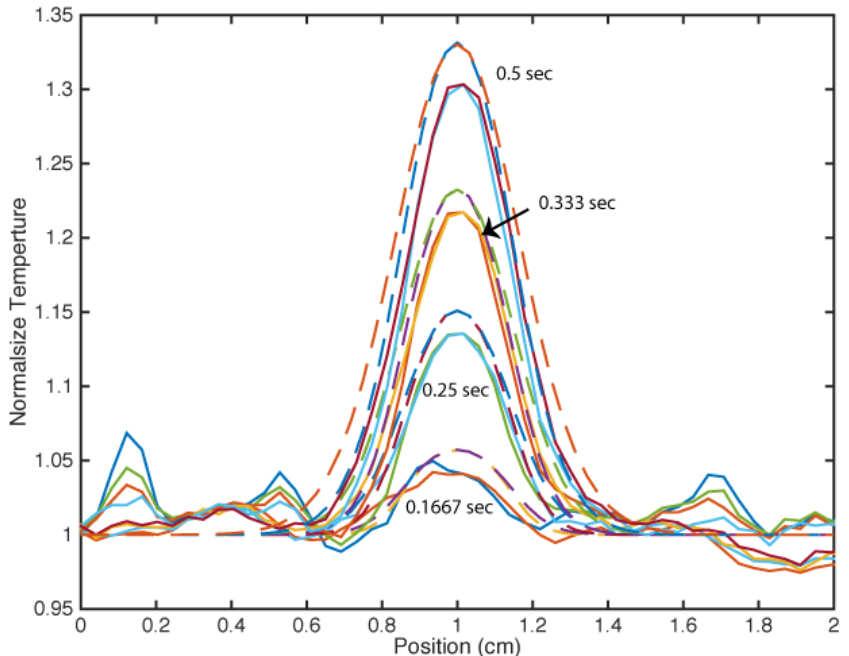

(b)

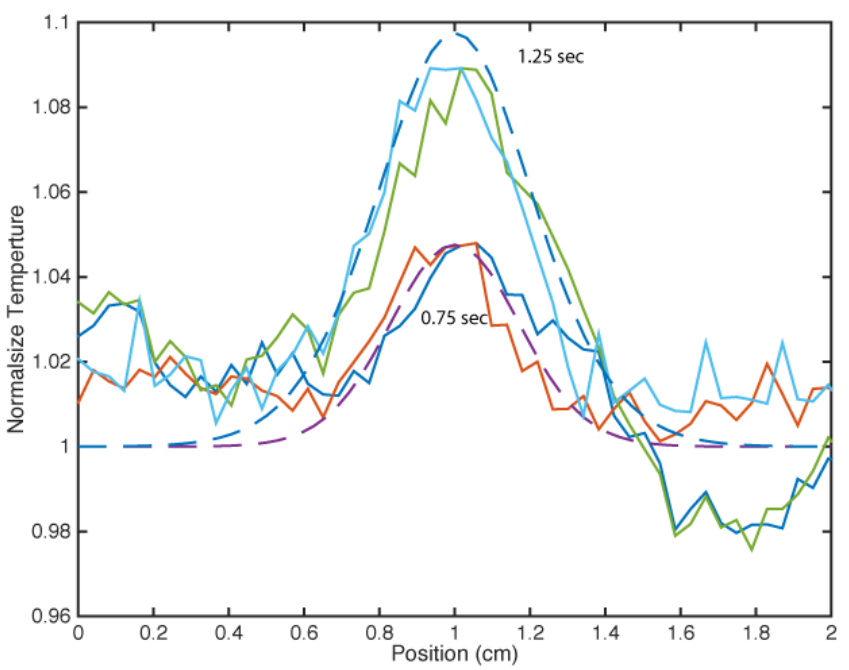

(d)

Figure 8. Comparison of quadrupole estimate of the thermal response of a flat bottom hole and the measured thermal response. Flat bottom hole characteristics are (a) $0.63 \mathrm{~cm}$ diameter $0.63 \mathrm{~cm}$, approximately $0.05 \mathrm{~cm}$ below the surface, (b) $0.32 \mathrm{~cm}$ diameter, approximately $0.05 \mathrm{~cm}$ below the surface, (c) $0.63 \mathrm{~cm}$ diameter $0.63 \mathrm{~cm}$, approximately $0.10 \mathrm{~cm}$ below the surface and (d) $0.32 \mathrm{~cm}$ diameter, approximately $0.10 \mathrm{~cm}$ below the surface. The experimental data are shown as solid lines and the simulation data are shown as dashed lines. The two different experimental curves are the horizontal and vertical profiles across the center of the indication. 
A comparison between the simulation results and the measured results is shown in Fig. 8. The times selected for comparison were based on the times when the measured responses were distinguishable when plotted together and are indicated on the plots. The data and the simulations were normalized be dividing by the mean value along the circumference of a $2 \mathrm{~cm}$ square centered on the indication. Both the horizontal and vertical profiles across the center of the indication are shown in the figure, however, for the experimental data the difference is not noticeable. Good agreement between the simulations and measurements was achieved by adjusting the depth in the simulations. For the four holes shown, the depths used were $0.0615 \mathrm{~cm}$ and 0.109 for the $0.32 \mathrm{~cm}$ diameter holes (holes 8 and 5 in the computed tomography image) and 0.055 and 0.105 for the $0.32 \mathrm{~cm}$ diameter holes (holes 7 and 4 in the computed tomography image). With the exception of the hole labeled 8 in Fig. $7(\mathrm{a})$, where a depth of $0.0615 \mathrm{~cm}$ was used, these depths were within the standard deviation of the computed tomography measurement. For this flat bottom hole, the thickness variation in the material above the hole results in a decrease in temperature at the center of the hole for early times. For shallow holes and early times, the simulation results are very sensitive to depth. The diameters of the circular delamination in the simulation were the values as shown in Tab. 1.

While delaminations are equivalent to flat bottom holes, the agreement between the simulations and experiment is very good. The times shown in the figure represent the early time response of both depths of holes. A significant difference between a flat bottom hole and a delamination is there is no head diffusion into the materials behind the hole surface as there is for a delamination. The good agreement may be an indication that sufficient time has not passed for significant heat flow around the flaw in the simulation.

\section{CONCLUSIONS}

A method for performing three dimensional simulations of the heat diffusion in a laminated composite is presented. The simulations were in good agreement with finite element method simulations of the same geometry and required one tenth of the time to compute. It was also shown that it is easy to insert realistically shaped flaws into the simulations. A comparison of the thermal response of flat bottom holes and simulations was shown to be in good agreement for early times.

\section{REFERENCES}

[1] Carslaw, H. S. and Jaeger, J. C., [Condtion of Heat in Solids], Clarendon Press, Oxford, 2 ed. (1986).

[2] Ozisike, N., [Finite Difference Methods in Heat Transfer], CRC Press, Inc, Boca Raton, Florida, 1 ed. (1994).

[3] Maldague, X. P., [Theory and Practice of Infrared Technology for Nondestructive Testing], John Wiley \& Sons, New York, New York, 1 ed. (2001).

[4] Krishnapillai, M., Jones, R., Marshall, I., Bannister, M., and Rajic, N., "Thermography as a tool for damage assessment," Composite Structures 67, 149-155 (2005).

[5] Krishnapillai, M., Jones, R., Marshall, I., Bannister, M., and Rajic, N., "NDTE using pulse thermography: Numerical modeling of composite subsurface defects," Composite Structures 75, 241-249 (2006).

[6] Winfree, W. P., Howell, P. A., Leckey, C. A., and Rogge, M. D., "Improved sizing of impact damage in composites based on thermographic response," in [SPIE Defense, Security, and Sensing], 87050V-87050V, International Society for Optics and Photonics (2013).

[7] Suša, M., Švaić, S., and Boras, I., "Pulse thermography applied on a complex structure sample: comparison and analysis of numerical and experimental results," in [IV Pan American Conference for Non Destructive Testing 2007], Hrvatska znanstvena bibliografija i MZOS-Svibor (2007).

[8] Plotnikov, Y. A. and Winfree, W. P., "Thermographic determination of delamination depth in composites," in [Thermosense XX], Proc. SPIE 3361, 331-339 (1998).

[9] Balageas, D. L., "Defense and illustration of time-resolved pulsed thermography for NDE," in [Thermosense: Thermal Infrared Applications XXXIII], Proc. SPIE 8013 (2011).

[10] Maillet, D., [Thermal quadrupoles: solving the heat equation through integral transforms], John Wiley \& Sons Inc, 1 ed. (2000). 
[11] Maillet, D., Houlbert, A., Didierjean, S., Lamine, A., and Degiovanni, A., "Non-destructive thermal evaluation of delamination in a laminate: Part I- Identification by measurement of thermal contrast," Composites Science and Technology 47, 137-153 (1993).

[12] Maillet, D., Batsale, J., Bendada, A., and Degiovanni, A., "Integral methods and non-destructive testing through stimulated infrared thermography," Revue Generale de Thermique 35(409), 5-13 (1996).

[13] Winfree, W. P. and Zalameda, J. N., "Thermographic determination of delamination depth in composites," in [Thermosense XXV], K. Elliot Cramer, X. P. M., ed., Proc. SPIE 5073, 363-373 (2003).

[14] Cramer, K. and Winfree, W., "The application of infrared thermographic inspection techniques to the space shuttle thermal protection system," Ensayos No Destructivos Y Estructurales , 227-233 (2005).

[15] Benítez, H., Ibarra-Castanedo, C., Bendada, A., Maldague, X., Loaiza, H., and Caicedo, E., "Modified differential absolute contrast using thermal quadrupoles for the nondestructive testing of finite thickness specimens by infrared thermography," in [Electrical and Computer Engineering, 2006. CCECE'06. Canadian Conference on], 1039-1042, IEEE (2006).

[16] Cramer, K. E. and Winfree, W. P., "Fixed eigenvector analysis of thermographic NDE data," in [SPIE Defense, Security, and Sensing], 80130T-80130T, International Society for Optics and Photonics (2011).

[17] Batsale, J., Maillet, D., and Degiovanni, A., "Extension de la méthode des quadripôles thermiques à l'aide de transformations intégralescalcul du transfert thermique au travers d'un défaut plan bidimensionnel," International Journal of Heat and Mass Transfer 37(1), 111-127 (1994).

[18] Bendada, A., "Approximate solutions to three-dimensional unsteady heat conduction through plane flaws within anisotropic media using a perturbation method," Modelling and Simulation in Materials Science and Engineering 10(6), 673 (2002).

[19] Bendada, A., Erchiqui, F., and Lamontagne, M., "Pulsed thermography in the evaluation of an aircraft composite using 3d thermal quadrupoles and mathematical perturbations," Inverse Problems 21(3), 857 (2005).

[20] Schroedera, J., Ahmedb, T., Chaudhryb, B., and Shepard, S., "Non-destructive testing of structural composites and adhesively bonded composite joints: pulsed thermography," Composites: Part A 33, 1511-1517 (2002).

[21] Shepard, S. M., Lhota, J. R., Rubadeux, B. A., Wang, D., and Ahmed, T., "Reconstruction and enhancement of active thermographic image sequences," Opt. Eng. 42, 1337-1342 (2003). 